\title{
A Proficient Adaptive K-means based Brain Tumor Segmentation and Detection Using Deep Learning Scheme with PSO
}

\author{
${ }^{1}$ Anitha T, ${ }^{1}$ Charlyn Pushpa Latha G, \& 2 Surendra Prasad M \\ ${ }^{1}$ Department of Computer Science \& Engineering, Saveetha School of Engineering, Saveetha Institute of Medical and \\ Technical Sciences (Deemed to be University), Chennai, Tamil Nadu, India. \\ ${ }^{2}$ Annapoorana Medical College and Hospitals. Salem - Kochi Highway, Kombadipatti, Tamil Nadu, India \\ *Corresponding Author: anitha.bioinformatics@gmail.com
}

Received: 10.08 .2020 , Revised: 14.11 .2020 , Accepted: 14.12 .2020 , Published: 22.12 .2020

DOI:

10.53409/mnaa.jcsit20201302

Abstract: Determining the size of the tumor is a significant obstacle in brain tumour preparation and objective assessment. Magnetic Resonance Imaging (MRI) is one of the non-invasive methods that has emanated without ionizing radiation as a front-line diagnostic method for brain tumour. Several approaches have been applied in modern years to segment MRI brain tumours automatically. These methods can be divided into two groups based on conventional learning, such as support vector machine (SVM) and random forest, respectively hand-crafted features and classifier method. However, after deciding hand-crafted features, it uses manually separated features and is given to classifiers as input. These are the time consuming activity, and their output is heavily dependent upon the experience of the operator. This research proposes fully automated detection of brain tumor using Convolutional Neural Network (CNN) to avoid this problem. It also uses brain image of high grade gilomas from the BRATS 2015 database. The suggested research performs brain tumor segmentation using clustering of k-means and patient survival rates are increased with this proposed early diagnosis of brain tumour using $\mathrm{CNN}$.

Keywords: Brain tumor, Computer-Aided Diagnosis, Deep Learning, Fusion Feature, Recurrent Extreme Learning Machine, Artificial Bee Colony.

\section{INTRODUCTION}

rain tumors are a heterogeneous group of neoplasms of the central nervous system that form within or adjacent to the brain. The incidence of tumors in India's central nervous system (CNS) ranges from 5 to 10 per 100,000 population with a rising trend [1]. Metastatic brain tumors arise when cancer travels to the brain situated in another organ of the body. Forty per cent of all cancers have spread to the brain. Recently computer-aided techniques have been investigated for the analysis and visualization of magnetic resonance (MR) images. A lot of researchers have focused on detecting and quantifying brain abnormalities. A significant step in this process is the automatic identification of the brain in head MR images. Another essential move for computer-aided research is the assurance of the data quality. The MR images involve unintended differences in intensity due to MRI scanner imperfections.

Removing or reducing such variations will increase automated analysis accuracy. This research introduces a new, fully automated method for the detection of intracranial boundary and intensity correction in head MR images. The intracrane boundary is the boundary between the intracranial cavity and the brain. It separates the brain correctly from other features within the head.

A new approach based on $\mathrm{CNN}$ is presented in this research which enables automatic detection and segmentation of brain tumors. The approach is based on the geodesic distance and covariance. The covariance approach for detecting central coordinates of irregular tissues is based on. Using adaptive clustering of $\mathrm{k}$ means, these coordinates are used for segmenting the brain tumor region. The ultimate goal is to retrieve the tumor attributes observed on the image in order to use them in the segmentation and classification stage. The present methods are based on MR images and have shown a better performance in biomedical image analysis.

The structuring of the rest of paper is as follows: In Section 2, discuss brain tumor detection methods. Section 3 describes the proposed 
methodology. Section 4 describes the results and discussion; in Section 5, conclude the paper and define the future work.

\section{RELATED WORK}

A hybrid brain tumor detection algorithm using statistical features in magnetic resonance images and a Fuzzy including kernel support vector machine (FSVM) classifier is proposed in [2]. Brain tumors are not diagnosed early and are not fully treated and patients may suffer permanent brain injury or death. Place and size of tumors are critical to effective treatment.

In the proposed system [3], the self-organizing map neural network initially trains the features extracted from the discrete wavelet transform blend wavelets and subsequently, the resulting filter factors are equipped by the K-nearest neighbor (KNN). The aim of the study in [4] is to use advanced image processing techniques and probabilistic neural network (PNN) method to detect and locate tumor areas in the brain.

An unsupervised tumor segmentation clustering method is proposed at [5]. In addition, a fused feature vector is used which is a mixture of the features of Gabor wavelet (GWF), oriented gradient histograms (HOG), local binary pattern (LBP) and fractal texture analysis based segmentation (SFTA). Random forest (RF) classifier is used to identify three sub-tumor regions such as the total, enhancing, and nonenhancing tumor.

In [6] Neural Network Ensemble is used to improve system accuracy. The Jaya algorithm used for segmentation, and also extraction of the abnormal brain section, requires less time to execute compared to the Particle Swarm Optimization and Genetic Algorithm used previously.

The fuzzy logic-based hybrid kernel is built in [7] and used to train the vector support system to automatically identify four different forms of brain tumors such as Meningioma, Glioma, Astrocytoma, and Metastases.

Gray-level co-occurrence matrix (GLCM) features, which segmentation of the DWT-based brain tumor area in [8] is used to minimize complexity and improve performance. In brain MRI images the probabilistic neural network classifier was used to detect tumor location.
The proposed method in [9] classifies brain MRI slices as regular or abnormal, using Gabor filter and supporting vector machines. The problem of auto classification of brain MRI slices as normal and abnormal has been identified to resolve numerous schemes, but precision, robustness, and optimization are still an open question..

\section{PROPOSED METHODOLOGY}

In this method, segmentation has been combined using adaptive clustering of K-means followed by detection as a classifier tool with convolutionary neural network (CNN). This adaptive k-means method incorporates the maximum connected domain algorithm for the adaptive determination of $\mathrm{K}$ values of the $\mathrm{K}$ means segmentation process. For a broad database, the segmented area from the adaptive $\mathrm{k}$-means was categorized as the normal and abnormal tumor cells of medical brain MRI images. In this work, the use of Particle Swarm Optimization (PSO) in Convolutionary Neural Networks (CNNs), which is one of the basic methods in deep learning, was proposed. Using PSO in the training phase helps to optimize the outcomes of solution vectors on CNN to increase the accuracy of tumor identification. The result includes the fact that it makes it easier for clinical experts to make a decision about diagnosis and even scanning with this proposed process. The work's principal contribution is as follows:

- Initially, pre-processing improves image quality by removing noise and the artifacts of the input MRI image, this study employs a median filtering technique to pre-process the image.

- Image segmentation is performed using adaptive k-means clustering to partition MRI image into mutually exclusive and exhausted regions, so that each area of interest is spatially contiguous and the pixels within the area are homogeneous to the predetermined rules.

- Finally, automatic brain tumor detection is achieved using the classification of Particle Swarm Optimization in the Convolutionary Neural Networks (CNN) as shown in Figure 1. 


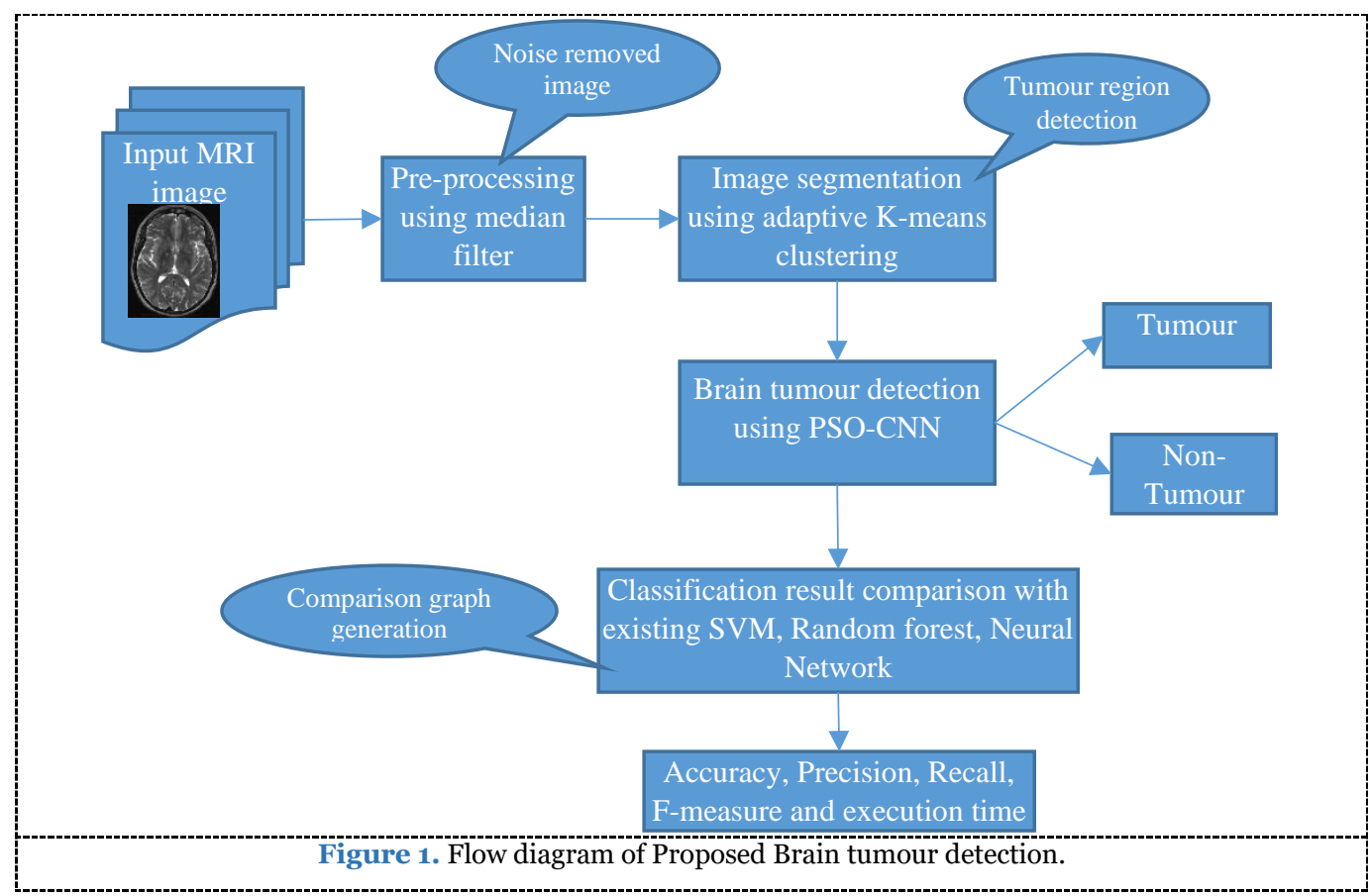

\subsection{Input MRI dataset}

The MRI data sets were collected from the public database (http:/adni.loni.usc.edu/) of the Harvard Medical School Architecture and Alzheimer's disease Neuroimaging Initiative (ADNI). A total of $100 \mathrm{MR}$ images were used for preparation, and $25 \mathrm{MR}$ images were used for research. The input MRI images will experience the gray image conversion process, tumor position detection and tumor segmentation may experience correlation computation. For relegation of benign and malignant tumors using GLCM (Grey Level Cooccurrence Matrix) technique a minimum of ten features are collected.

\subsection{Image Pre-processing Using Median Filter}

The image processing is really complicated. Before any picture is processed, removing unnecessary objects that it may contain is quite important. The picture can be processed successfully after extracting unwanted artefacts. Image PreProcessing is the key step to image processing. It includes processes such as grayscale image conversion, noise reduction, and image restoration. The most popular method of pre-processing is conversion to gray scale image. After the image is converted to grayscale, then using Median Filter methods to eliminate excess noise. This most famous technique used during the reduction of noise. It is a filtering method called "non-linear." It has been used to suppress the grayscale picture created by salt and pepper noise. Median filter is based on average pixel size, and therefore retains the edges and boundaries [10].

\section{3. $\quad$ Image segmentation using adaptive $K$ - means clustering}

In this method, brain image segmentation using Kmeans clustering technique to segment the tumor from the image as a contour to Variational Level sets, which in turn performs tumor boundary removal. K-Means is one of the unsupervised cluster learning methods. Clustering the image groupes the pixels by the same characteristics [11].

1. Initialize $\mathrm{k}$ as the cluster value no.

2. Always choose k-cluster centers at random

3. Determine cluster mean or centre

4. Compute the difference between pixels and centers of each cluster

5. If the distance to the center is near then transfer to that cluster.

6. Move to next cluster otherwise.

7. Re-evaluate the centre.

8. Repeat the cycle before the core comes in.

\subsection{Feature Extraction using GLCM}

Features of the image were extracted using the Gray Level Co-occurrence Matrix (GLCM). In this feature extraction technique, initially GLCM of the image was calculated then the features based on the GLCM were extracted. The extracted features include contrast, correlation, energy, homogeneity, mean, standard deviation, entropy, skewness, kurtosis, inverse difference moment. 


\subsection{Brain tumour detection using PSO-CNN}

In this study, we perform a combination of biologically inspired Particle Swarm Optimization and Convolutional Neural Network as a classifier tool to improve diagnostic accuracy. The cause of this study is to extract information from the segmented tumor region and classify healthy and infected tumor tissues for a large database of medical images. Our results lead to conclude that the proposed method is suitable to integrate clinical decision support systems for primary screening and diagnosis by the radiologists or clinical experts.

Convolutional Neural Networks (CNN) are one of the most used neural networks in the present time. Its applications are extremely varied. Most recently they have been proving helpful with deep learning, as well. Since it is growing in more convoluted domains, its training complexity is also increasing. To tackle this problem, many hybrid algorithms have been implemented. In this paper, Particle Swarm Optimization (PSO) is used to reduce the overall complexity of the algorithm. The hybrid of PSO used with CNN decreases the required number of epochs for training and the dependency on GPU system. The algorithm so designed is capable of achieving $3-4 \%$ increase in accuracy with lesser number of epochs. The advantage of which is decreased hardware requirements for training of CNNs. The hybrid training algorithm is also capable of overcoming the local minima problem of the regular backpropagation training methodology.

Generally, the process of the proposed method consists of several steps as shown below:

1) The first step is initializing the learning rate of the CNNs with the value is 1 based on the experiment. Batch size of $\mathrm{CNNs}$ is 50, the number of CNNs epoch in the range of 1 to 4 , PSO iteration is 10. The convergence status of PSO is used to check the convergences of PSO, if the error value has not changed for three iterations, then the PSO is considered as convergent;

2) After setting up the experiment, the next step is run CNNs training process, where the tumour is detected from the feature extraction process. The result of CNNs is vector output that will be optimizing using PSO algorithm. PSO optimization in this study serves to make the value of loss function on $\mathrm{CNN}$ becomes minimal;

3) The output vector will be update if the solution of swarm has less error compare with old vector output;

4) The PSO will run as long as the iteration number of PSO and the convergence solution have not fulfilled;

5) After the CNN Training, the model will be tested with testing data that consist of MRI brain image data;
6) The result of $\mathrm{CNN}$ test is accuracy of $\mathrm{CNN}$, it represent how precise of the $\mathrm{CNN}$ model can predict the actual value of testing dataset.

\section{EXPERIMENTAL RESULTS AND DISCUSSION}

The performance of the proposed PSO-CNN is evaluated in this section, and the performance results are compared with existing FSVM [1], KNN [2] and PNN [3] image compression schemes. The facts given below show that the device proposed has achieved better performance in terms of precision, f-measurement, recall and accuracy.

Precision: It reflects the proportion of positive samples correctly classified as expected in equation (6):

$$
\text { Precision }=\frac{T P}{F P+T P}
$$

Recall: The recall of a classifier reflects the positive samples properly assigned to the total number of positive samples and is calculated as in equation (7):

$$
\text { Recall }=\frac{T P}{T P+F N}
$$

F-measure: this is also referred to as F 1-score, and as in equation (8) is the harmonic mean of precision and recall:

(8):

$$
F-\text { measure }=\frac{2 *(\text { Recall } * \text { Precision })}{(\text { Recall }+ \text { Precision })}
$$

Accuracy: This is one of the most frequently used performance classification measures and is defined as a ratio between the correctly classified samples and the total number of samples as in equation (9):

$$
\text { Accuracy }=\frac{T P+T N}{T P+T N+F P+F N}
$$

Where true positive (TP) samples are properly classified as natural, false positive (FP) samples are incorrectly classified as irregular, True negative (TN) samples are properly classified as irregular, and false negatives (FN) are incorrectly classified as natural.

\subsection{Precision Rate comparison}

From the above Figure 4, the graph shows how accurate the number of images in the specified datasets is compared. These methods are implemented as FSVM, KNN, PNN, and PSO-CNN. When the number of records increases according to the precision value. 


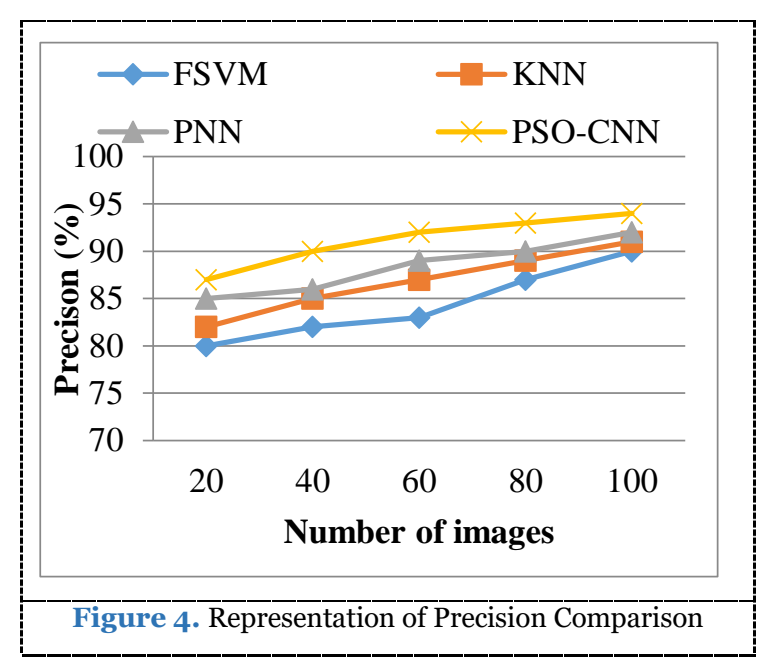

From this graph, it is learned that the proposed PSO-CNN offers 94\% higher precision than previous methods that yield better results in the classification of Brain tumour mass due to prior segmentation of the Brain tumour mass using kmeans technique. The numerical results of Precision Comparison is shown in Table 1.

Table 1. The numerical results of Precision Comparison

\begin{tabular}{ccccc}
\hline No. of images & FSVM & KNN & PNN & PSO-CNN \\
\hline 20 & 80 & 82 & 85 & 87 \\
\hline 40 & 82 & 85 & 86 & 90 \\
\hline 60 & 83 & 87 & 89 & 92 \\
\hline 80 & 87 & 89 & 90 & 93 \\
\hline 100 & 90 & 91 & 92 & 94 \\
\hline
\end{tabular}

\subsection{Recall comparison}

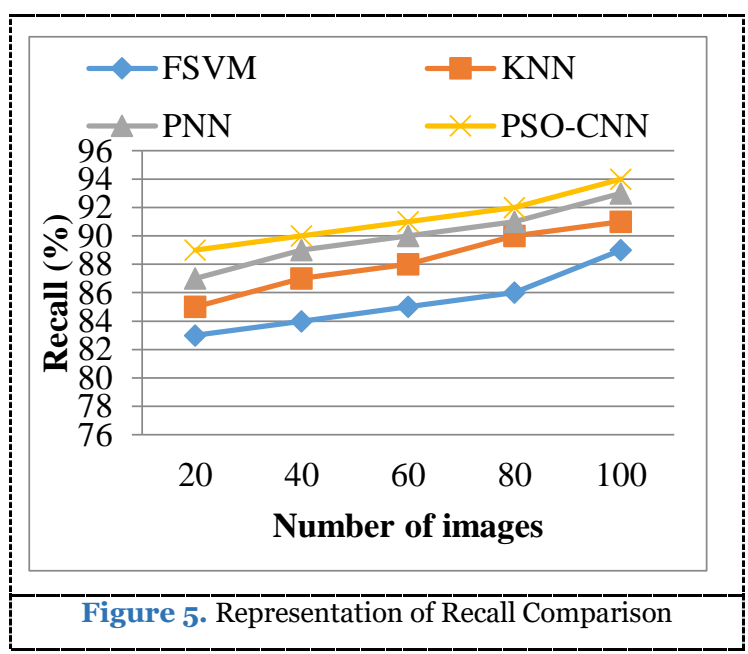

From the above Figure 5 the graph illustrates the recall relation for the number of images in the listed datasets. These methods are implemented as FSVM, $\mathrm{KNN}, \mathrm{PNN}$, and PSO-CNN. Increasing the number of photographs often increases the correct value for the recall. Through this graph, it is discovered that the current PSO-CNN offers recall 94\% higher than previous methods. The explanation for this is that the PSO-CNN extracts the features directly which will enhance the Brain tumour classification tests. The numerical results of Recall Comparison is shown in Table 2.

Table 2. The numerical results of Recall Comparison

\begin{tabular}{ccccc}
\hline No. of images & FSVM & KNN & PNN & PSO-CNN \\
\hline 20 & 83 & 85 & 87 & 89 \\
\hline 40 & 84 & 87 & 89 & 90 \\
\hline 60 & 85 & 88 & 90 & 91 \\
\hline 80 & 86 & 90 & 91 & 92 \\
\hline 100 & 89 & 91 & 93 & 94 \\
\hline
\end{tabular}

\subsection{F-measure Rate comparison}

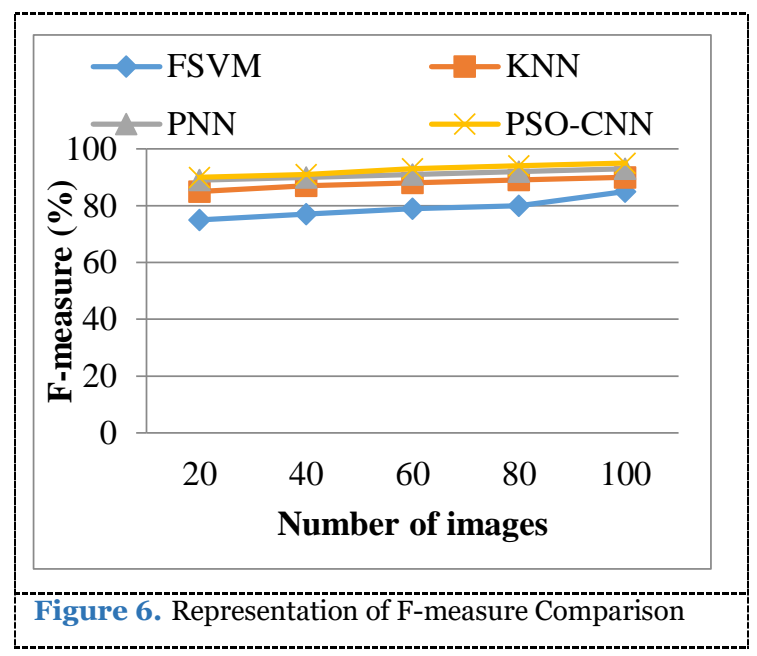

From the above Figure 6, the graph explains the fmeasure relation for the number of images in the given datasets. These methods are implemented as FSVM, KNN, PNN, and PSO-CNN. When the number of data is increased, and the f-measure value is increased accordingly. From this graph it is learned that the proposed PSO-CNN offers 95\% higher f-measurement than previous methods.

Therefore the proposed PSO-CNN algorithm is stronger than the current algorithms in terms of better performance of classifying Brain tumour. The numerical results of F-measure Comparison is shown in Table 3.

Table 3. The numerical results of F-measure Comparison

\begin{tabular}{ccccc}
\hline No.of images & FSVM & KNN & PNN & PSO-CNN \\
\hline 20 & 75 & 85 & 89 & 90 \\
\hline 40 & 77 & 87 & 90 & 91 \\
\hline 60 & 79 & 88 & 91 & 93 \\
\hline 80 & 80 & 89 & 92 & 94 \\
\hline 100 & 85 & 90 & 93 & 95 \\
\hline
\end{tabular}




\subsection{Accuracy comparison}

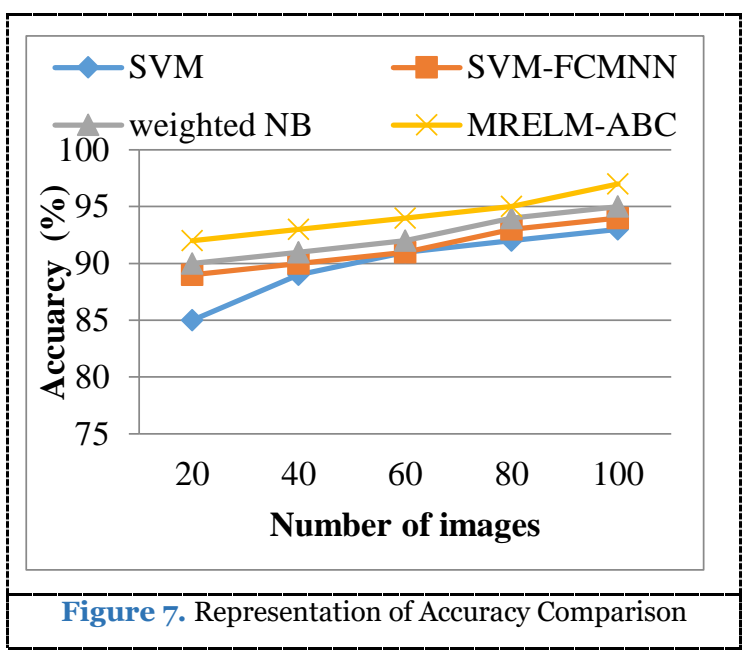

From the above Figure 7 the diagram illustrates the processing time relation for the number of images in the specified datasets. These methods are implemented as FSVM, KNN, PNN, and PSO-CNN. From this graph it is known that the proposed PSO$\mathrm{CNN}$ algorithm is higher than the existing algorithms with a high precision rate of $97 \%$ in terms of better template matching results. This is due to the automatic extraction of the features using $\mathrm{CNN}$ in the PSO-CNN classification algorithm, which increases the classification precision resulting in Brain tumour. The numerical results of Accuracy Comparison is shown in Table 4.

Table 4. The numerical results of Accuracy Comparison

\begin{tabular}{ccccc}
\hline No.of images & FSVM & KNN & PNN & PSO-CNN \\
\hline 20 & 85 & 89 & 90 & 92 \\
\hline 40 & 89 & 90 & 91 & 93 \\
\hline 60 & 91 & 91 & 92 & 94 \\
\hline 80 & 92 & 93 & 94 & 95 \\
\hline 100 & 93 & 94 & 95 & 97 \\
\hline V. CONCLUSION AND FUTURE \\
WORK
\end{tabular}

In this work, a method to extract brain tumor from 2D Magnetic Resonance brain Images (MRI) by adaptive k-means clustering algorithm is proposed which was followed by traditional classifiers and convolutional neural network. In this work, we propose a novel algorithm to search for deep convolutional neural networks (CNNs) architectures based on particle swarm optimization (PSO-CNN). A novel directly encoding strategy is also proposed in which a CNN architecture is divided into two blocks: one block contains only convolutional and pooling layers, while the other contains only fully connected layers. This encoding strategy allows for variable length CNN architectures to be compared and combined using an almost standard PSO algorithm. Our results show that PSO-CNN can quickly find an optimized $\mathrm{CNN}$ architecture for any given dataset. With only 30 particles and 20 iterations, the algorithm finds models capable of achieving test errors comparable to those designs exploiting more complex and complicated architectures. From the experimental results, we can conclude that psoCNN would be able to find even better architectures if more computational power is available for finding MRI brain tumor. For future works, we will expand the proposed PSO-CNN to find to include ResNets and DenseNets architectures as well, which will further increase the classification accuracy of the possible solutions.

\section{REFERENCES}

[1]. Deshpande, R. P., Babu, D., Panigrahi, M., Sekhar, Y. C., \& Babu, P. P. (2016). Brain tumors incidences and a retrospective clinical analysis from a tertiary hospital in India. Journal of neuro-oncology, 129(2), 383-387.

[2]. Jayachandran, A., \& Sundararaj, G. K. (2015). Abnormality segmentation and classification of multi-class brain tumor in MR images using fuzzy logic-based hybrid kernel SVM. International Journal of Fuzzy Systems, 17(3), 434443.

[3]. Anitha, V., \& Murugavalli, S. J. I. C. V. (2016). Brain tumour classification using two-tier classifier with adaptive segmentation technique. IET computer vision, 1O(1), 9-17.

[4]. Ural, B. (2018). A computer-based brain tumor detection approach with advanced image processing and probabilistic neural network methods. Journal of Medical and Biological Engineering, 38(6), 867-879.

[5]. Amin, J., Sharif, M., Raza, M., \& Yasmin, M. (2018). Detection of brain tumor based on features fusion and machine learning. Journal of Ambient Intelligence and Humanized Computing, 1-17.

[6]. Kaur, K., Walia, G. K., \& Kaur, J. (2018). Neural Network Ensemble and Jaya Algorithm Based Diagnosis of Brain Tumor Using MRI Images. Journal of the Institution of engineers (India): Series B, 99(5), 509-517.

[7]. Jayachandran, A., \& Sundararaj, G. K. (2015). Abnormality segmentation and classification of multi-class brain tumor in MR images using fuzzy logic-based hybrid kernel SVM. International Journal of Fuzzy Systems, 17(3), 434443 .

[8]. Shree, N. V., \& Kumar, T. N. R. (2018). Identification and classification of brain tumor MRI images with feature extraction using DWT and probabilistic neural network. Brain informatics, 5(1), 23-30.

[9]. Gilanie, G., Bajwa, U. I., Waraich, M. M., Habib, Z., Ullah, H., \& Nasir, M. (2018). Classification of normal and abnormal brain MRI slices using Gabor texture and support vector machines. Signal, Image and Video Processing, 12(3), 479-487.

[10]. Roy, S., \& Bandyopadhyay, S. K. (2012). Detection and Quantification of Brain Tumor from MRI of Brain and it's Symmetric Analysis. International Journal of Information and Communication Technology Research, 2(6).

[11]. Moftah, H. M., Azar, A. T., Al-Shammari, E. T., Ghali, N. I., Hassanien, A. E., \& Shoman, M. (2014). Adaptive k-means clustering algorithm for MR breast image segmentation. Neural Computing and Applications, 24(78), 1917-1928. 